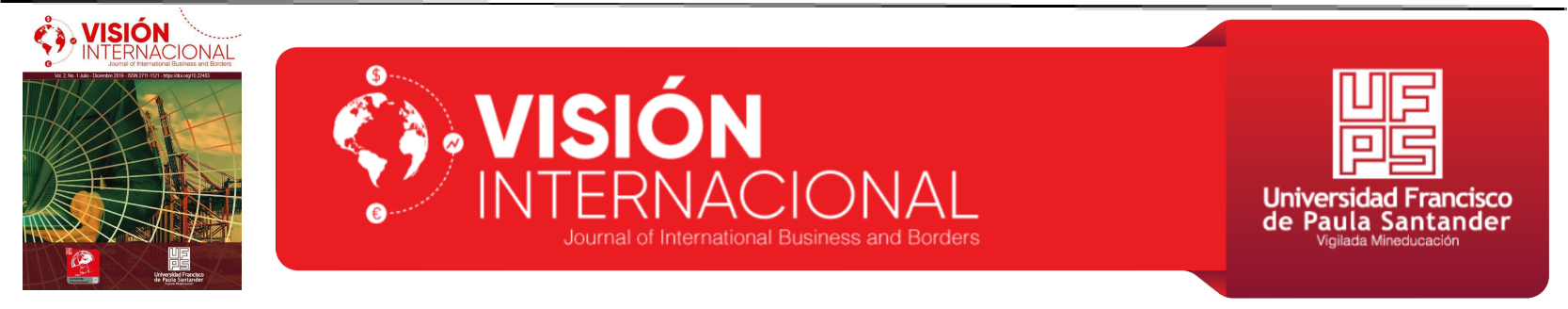

\title{
Análisis de la problemática comercial que deja la separación de la península coreana
}

\author{
Analysis of the commercial problem caused by the separation of the Korean península \\ Lissette Gabriela Maldonado Niño ${ }^{a *}$, Sirley Oliveros Meza ${ }^{b}$, Jose Javier Rojas Laguado ${ }^{c}$ \\ $a^{*}$ Magister en administración de empresas ,Lissettegabrielamn@ufps.edu.co, https://orcid.org/0000-0002-4920-3955,Universidad Francisco de Paula \\ Santander, Cúcuta, Colombia. \\ ${ }^{b}$ Profesional en Comercio Internacional, vienasirleyom@ufps.edu.co, Universidad Francisco de Paula Santander, Cúcuta, Colombia. \\ ${ }^{c}$ Profesional en Comercio Internacional, josejavierrl@ufps.edu.co, Universidad Francisco de Paula Santander, Cúcuta, Colombia
}

Cómo citar: Maldonado-Niño, L.G. Oliveros-Meza, V.S. Rojas-Laguado, J.J. (2019) Análisis de la problemática comercial que deja la separación de la península coreana. Revista Visión Internacional, 2(5), 32-39

Recibido: Enero 10 de 2019; Aceptado: Marzo 202019

\begin{tabular}{ll}
\hline RESUMEN \\
\hline Palabras clave: & $\begin{array}{l}\text { Este artículo tiene por objeto dar a conocer y entender el origen de la problemática } \\
\text { de la península coreana, mostrando así mismo los factores sociales y comerciales }\end{array}$ \\
$\begin{array}{l}\text { Problematica } \\
\text { Relaciones internacionales }\end{array}$ & $\begin{array}{l}\text { se han tejado la división de esta nación, por otra parte muestra las afectaciones que } \\
\text { conociendo las relaciones internacionales mostrando así mismo la intervención que } \\
\text { Inversión extranjera }\end{array}$ \\
$\begin{array}{l}\text { Reunificacion } \\
\text { huclearización }\end{array}$ & $\begin{array}{l}\text { muestra los logros que se han obtenido en la reunificación de las coreas dejando a un } \\
\text { lado los temas de la nuclearización. }\end{array}$ \\
\hline & ABSTRACT \\
\hline Keywords: & $\begin{array}{l}\text { This article aims to know and understand the origin of the problem of the Korean } \\
\text { peninsula, also showing the social and commercial factors that have left the division } \\
\text { of this nation, on the other hand shows the effects that have been had at the level of }\end{array}$ \\
Problems, & education and with the deserters of these nations; knowing the international relations \\
International relations, & showing also the intervention that the powers in the conflict have had, the foreign \\
Foreign investment, & investment; Finally, it shows the achievements that have been obtained in the \\
reunification, & reunification of Korea, leaving aside nuclearization. \\
nuclearization. &
\end{tabular}

\section{Introducción}

En el siguiente artículo se quiere definir el conflicto que hay dentro de la península Coreana, sus orígenes y causas además de los factores externos que afecta esta guerra de más de 40 años. Por medio de escritores propios de la península y algunos pensadores latinoamericanos y europeos veremos los distintos puntos de vista ya sean políticos, económicos o culturales los cuales tienen relevancia en el diario vivir de los habitantes de Corea del norte y Corea del sur, las relaciones internacionales que desprenden cada país y como se defienden en el ámbito comercial.

*Autor de Correspondencia.

E-mail: Lissettegabrielamn@ufps.edu.co

(Lissette Gabriela Maldonado Niño)

(c) $(i)$

Peer review is the responsibility of the Universidad Francisco de Paula Santander

This is an article under the license CC BY-ND 
Los factores de salud y conducta hacia las víctimas del conflicto o desertores y como una aguda crisis está acercando la posibilidad de una reunificación dentro de la península.

\section{Método}

Es este artículo se realizó un tipo de investigación cualitativa, utilizando un método descriptivo y documental, dado que para la elaboración se obtuvo por muestra no probabilística de manera aleatoria con una selección de 21 artículos, los cuales se utilizaron bajo la perspectiva de la inclusión y la exclusión todo con el objetivo de incrementar los conocimientos relacionados sobre el tema de la problemática comercial que deja la separación de la península coreana, obtenidos de varias bases de datos de investigación científica como son Dialnet, Redalyc, Scielo, Science Direct, Google Académico, y Revistas Indexadas.

Origen de la problemática

Para dar inicio se van a mencionar algunos factores de la península coreana como lo exponen en su libro, conflicto de Corea. Lafuente, E \& Fernández, L. (2013)

La cual cuenta con una longitud aproximadamente de 900 y anchura de 200 para una superficie de 219 mil kilómetros cuadrados. Está bañada al oeste por el mar Amarillo, al este por el mar del Japón (o, como se le denomina en Corea, el mar del Este) y al sur por los estrechos de Chejú y de Corea. (p.15)

A lo largo de la historia la Península Coreana ha tenido que ver las guerras de sus más grandes vecinos, Japón, China y Rusia, después de la separación de los dos estados se generó un desequilibrio demográfico dado a un efecto por el abandono de una gran parte de la población del norte estos dos mundos están separados por el paralelo 38 aunque tienen raíces históricas, étnicas y lingüísticas, tienen realidades opuestas que son políticas y económicas ya que una de las dos es comunista y la otra es democrática. Mora, J.P (1998).

Guerra de corea y creación de dos bloques
Para dar continuidad con la investigación del libro conflicto de corea, se hace importante el aporte que los autores Lafuente, E. \& Fernández, L. (2013) afirman:

Los coreanos por más de mil años compartieron una cultura homogénea hasta el año de 1905 donde los japoneses se declararon su protector imperial; pero esta tuvo lugar a la administración japonesa hasta 1945, después de la Segunda Guerra Mundial la península coreana se dividió en dos dando así en la zona del norte que era gobernada por la Unión Soviética a la República de Corea (ROK) y en la zona del sur por los Estados Unidos a la República Popular Democrática de Corea (DPRK). En 1953 Corea del Sur siempre ha proclamado la Unificación de Corea como un solo estado, sin embargo esta nunca ha aceptado a Corea del Norte como un estado independiente. A su vez Corea es el único país dividido por la doctrina mundial, siendo así los que soportaron la horrible guerra entre los Estados Unidos y la ex -Unión Soviética. (p. 30)

\section{La internacionalización del conflicto}

Todo comienza cuando Estados Unidos y China determinan interponerse generando así una posible Tercera Guerra Mundial; para Estados Unidos el participar en el conflicto era conservar su reputación poderío a nivel mundial. Astor Molero, P. (2015).

\section{Economía}

Tras el final de la guerra en 1965 Corea del Norte tenía una economía que doblaba la renta per cápita siendo así una economía más sólida que la de Corea de Sur, rápidamente después de esto la economía agraria de Corea del Sur sufrió un cambio convirtiéndose así en una economía industrializada dándole un aumento al producto interno bruto. Chiñas, C. G. (2003).

El investigador (Giné, 2008) ha señalado que Corea del Sur ha experimentado varios cambios a nivel político y social. En 2008 ocupaba el puesto número doce de la economía mundial y la en hacia la cuarta después de Japón, China y 
India. Siendo la número once en las exportaciones y en las importaciones la número trece. Esto se debe a la formación de las nuevas generaciones y al desembolso en el tema de la investigación y el desarrollo del país a nivel nacional e internacional.

Por otro lado, su mercado laboral tiende a caracterizarse con un quebranto en la tasa de desempleo ya que se encuentra entre el $3.5 \%$ y el $4.5 \%$, aunque por la población más joven con un estimado hasta los 24 años se eleva en un $8 \%$. Por parte de áreas industriales se puede evidenciar que siete de las diez empresas más importantes a nivel mundial se ubican en la construcción naval dedicadas a esta producción, en la industria siderúrgica contrario a que no cuenta con recursos de carbón y hierro es uno de los principales productores de acero a nivel mundial otras industrias son la petroquímica y construcción, automovilística y electrónica.

Por parte de Corea del Norte está siempre se ha caracterizado por la planificación y la autarquía. A partir de los años sesenta desarrollo una industria siderúrgica notable en la producción de maquina pesada, acero y también en la construcción naval ya que siempre cuenta con recursos minerales de plomo, níquel, magnesia y mercurio. No obstante Corea del Norte no se dedicaba mucho a los bienes de consumo, porque tenía un eminente aumento en la industria pesada. Sin embargo en los años noventa tras la derrota de la Unión Soviética se detuvieron en su mayoría parte de la producción de la industria ya que este era su esencial proveedor de combustible dejando a Corea del Norte en una crisis económica, sobre todo por el absurdo porcentaje del Producto Interno Bruto en gastos de defensa. Chiñas, C. G. (2003).

\section{Realidad intercoreana}

Aunque Corea del Sur y Corea del Norte han hecho varios esfuerzos para arreglar su relación hay algunos muchos impedimentos en medio de estos, en 1961 la Casa Azul del presidente fue atacada por los llamados bandidos rojos, 1968 en Corea del Sur dos ciudades tuvieron desacuerdos, 1976 unos soldados norcoreanos asesinaron a otros del Sur en la zona desmilitarizada (DMZ) posteriormente a la declaración de paz, 1985 se dio el encuentro de las familias separadas, 1987 se dio la explosión del avión de KAL (Korean Airlines, la línea surcoreana) bajo las órdenes de Corea del Norte.

\section{Organización política}

Actualmente Corea del sur está dentro de un marco democrático que hacía mucho tiempo no se veía en un gobierno donde se implementaron una serie de cambios en los cuales está la libertad de prensa y la libre elección del mandatario por parte de los ciudadanos incluso después de muchos años en 1992 fue electo el primer presidente civil, después de muchos años siendo militares los únicos para este puesto. A tal medida que incluso, Park Geun-Hye se convirtió en la primera presidenta mujer desde 2013 hasta su destitución en el año de 2017; siendo esto un revuelo pues es la primera vez que un mandatario es destituido de tal manera, principalmente tráfico de influencias y fraude por terceros, donde en su campaña luchó por conseguir una mayor participación de la mujer en una sociedad a cargo de los hombres. Más allá de esto, se puede decir que Corea del sur es una economía abierta y que a través de sus distintos gobernantes se han aplicado una serie de leyes de liberación de mercados, internacionalización de la economía. Shin, H., \& Stevens, Q. (2013).

En Corea del Norte se desconocen los datos reales económicos, poseen un sistema político socialista de contraparte comunista, un sistema muy cerrado el cual es considerado unos de los menos viables y cambiantes del mundo donde no hay un sistema democrático $\mathrm{y}$ todos sus gobernantes han alcanzo tal nivel de "reverencia" y "alabanza" que se les ha llamado supremos lideres a los dictadores. El presidente actual Kim-Jong- Un, destina la gran mayoría de su presupuesto al ejército nacional de corea el cual es que gran beneficiado en un país pobre sin industria y que con agricultura se va sosteniendo, tampoco hay libertad de prensa o pensamiento en esta península.Yang, E. (2012) 
El factor nuclear sin duda alguna es lo que más llama la atención de corea del norte y cuántas veces ha sido sancionada por diferentes organismos por sus pruebas nucleares que igual han sido controladas y en su propio territorio. Esto es contradictorio para la comunidad internacional ya que algunos mandatarios creen que esas pruebas nucleares sólo se hicieron para llamar la atención del contexto mundial y no para una posible guerra como dicen. Park, S., Lee, M., Park, S. J., \& Lee, M. G. (2018).

\section{Educación}

En el siguiente informe se habla sobre el rol de la política educacional en el conflicto de las dos Coreas y como ha conllevado a través de los años; Antes de la invasión japonesa las dos Coreas compartían un legado histórico cultural, existen muchos factores de separación, pero uno de los más relevantes son los del contexto nacional e internacional. Los habitantes del Norte tienen una visión muy diferente. A lo largo de la historia se han implementado una serie de políticas educacionales iniciando desde los años 60 con la llamada: "educación anticomunista" que era netamente en contra de la ideología de los norcoreanos, existieron varias reformas y luego pasó a ser la "educación para la unificación”. Min, W. (2004).

La educación coreana es algo compleja, para los habitantes del sur la educación para la unificación es una manera de preparar a sus habitantes para cualquier avance industrial, y es que corea del sur de por sí y su milagro económico se dio porque preparo a sus habitantes desde corta edad para el mundo laboral dándole las herramientas necesarias para defenderse y haciendo de ellos unos buenos administradores y teniendo una visión de valor. Aquí una diferencia sobre lo que es la educación para los del sur y del norte, donde para los norcoreanos es urgente que empiecen a formar parte de la educación para la unificación, para ello es importante la cooperación y el diálogo lo cual es la base de la educación. Min, W. (2004).
Se puede considerar que la unificación de las dos coreas está lejos, pero con estas reformas educacionales se quiere llegar a eliminar esas barreras culturales que tal vez por culpa de la internacionalización aún persisten al igual que puede ser por el factor nuclear donde el vecino del norte tiene un potencial destacado. La unificación podría estar cerca cuando todos estos temas de internacionalización no sean tan relevantes para la península coreana, para lograrla es de vital importancia la unión de este pueblo. Min, W. (2004).

En la presente investigación se dan a conocer los resultados de cómo los estudiantes universitarios de Corea del Norte interactúan con los estudiantes de Corea del Sur. Un ensayo para determinar la convivencia y las relaciones interpersonales entre ambos países en un ambiente y contexto académico.

La adaptación de los estudiantes de corea del norte es algo arduo, convivir con estudiantes del sur sobre todo en las clases de idiomas, en especial el INGLES, donde esta lengua significa para los norcoreanos la clave del "Éxito". Existen varios factores del por qué los estudiantes del norte no son tan buenos para la clase de idiomas. Poseen una condición de refugiados, donde algunos han tenido que atravesar esa frontera solos o solo cuentan con un miembro de su familia del lado del sur, por ende la rotación de escuelas es algo común para ellos. No recibir la educación necesaria para desenvolverse mejor en las clases de lenguaje. Esto se evidencia cuando en los ejercicios practicados entre dos estudiantes, el norcoreano siempre es más cerrado en la conversación y accede a lo que el estudiante de corea del sur le plantea. Su acento ha sido tema de conversación y hasta de burla en algunos repertorios de opinión pública. El estudiante norcoreano por esto es tan condescendiente y es más cerrado. La brecha que existe entre los estudiantes del norte y del sur es grande, abarcando desde sus gustos hasta sus pensamientos y puntos de opinión. Pero solo basta con hablar con los estudiantes del sur los cuales piensan que son tan iguales como ellos, la única diferencia es su acento, pero 
tienen sus mismas ganas de ser exitosos y salir adelante. Lee, M. W., \& Ahn, S. H. G. (2016).

Existió un acuerdo incondicional entre los estudiantes del norte y del sur donde estos no se reprochaban ni juzgaban a la hora de conversar en inglés, pero lo que si llamo la atención fue la exclusión del tema de su cultura y tópicos referentes a su país donde los estudiantes de corea del norte respondieron que no debe haber relevancia en eso estando ellos en corea del sur por lo consiguiente no prefieren hablar de eso. Lee, M. W., \& Ahn, S. H. G. (2016).

\section{Desertores}

Un tema de discusión a lo largo de la historia es la identidad nacional, donde en Corea la cual se creía que era una misma nación con dos países diferentes dentro de ella nos damos cuenta que no es así, Ha existido una homogeneidad de Nación, pero esto ha causado a su vez una discriminación hacia los habitantes del norte y los trabajadores o inmigrantes provenientes de la China.

Sí, es verdad que la península coreana comparte muchas tradiciones, su etnia y su cultura la cual es parte fundamental de su existir, pero está tan marcado el nacionalismo algunas veces que ya sea por el tono de piel o el acento existe una xenofobia y discriminación al norcoreano el cual ha tenido que sobrellevar una vida totalmente diferente en los aspectos políticos y sociales y se encuentra un poco marginado de la sociedad en un ambiente comunista que lo limita. La identidad política también es un factor de discusión para los habitantes del norte y del sur, para conocer la actitud hacia los desertores por parte de los habitantes de corea del sur hacia los del norte se ve reflejada una encuesta donde la mayoría cree que los norcoreanos son una comunidad marginada y por ende debe recibir apoyo tal y como fuese un país en vía de desarrollo y es que no hace menos de unas 4 décadas estaban compartiendo historia política luego vino la separación forzada pero en el fondo son una sola península con muchos factores desnivelados y con diferente calidad de vida.
Para los ciudadanos de la capital Pyongyang que han podido escapar y llegar al otro lado de la frontera incluso a otro continente es un escollo. Relatar las múltiples prohibiciones por parte del régimen socio-comunista y las condiciones establecidas por el mismo gobierno de cómo vivir, actuar y pensar.

La identidad política supera la homogeneidad étnica o eso es lo que se entiende cuando la actitud de los habitantes de corea del sur no es tan buena con los inmigrantes norcoreanos sobre todo los que provienen de China para trabajar. Los habitantes del norte a raíz de la crisis de hambruna en la década de los 90 tuvieron que traspasar su frontera en busca de una nueva vida, de nuevas oportunidades. A pesar de esto las cosas han venido cambiando $y$ en varias encuestas realizadas a los surcoreanos afirman que se debe aumentar el número de desertores norcoreanos, es decir ahora se sienten más cercanos a ellos.

Para Corea del sur el tema de la identidad política es relevante porque con esto difieren que decisiones y que pensamientos son los correctos, el orgullo nacional y el nacionalismo a pesar de ser una de las regiones más compactas (homogéneas) del mundo.

\section{Panorama de las relaciones internacionales}

Para entender como son las relaciones internacionales en la península coreana es necesario conocer la serie de cambios que ha habido desde la llegada del presidente Kim- Jong Un el predecesor de su padre donde ha intervenido $\mathrm{y}$ es el que inicialmente freno los intentos de unificación y tomo de lleno los proyectos nucleares dándole mayor importancia.

Corea del sur por lo contrario es una región que le apuesta a la innovación, es una de las grandes economías del mundo y posee un excelente plan de mejoramiento continuo además de un gobierno de apoyo con un plan de contingencia hasta el año 2030. Es un país motivado con una excelente mano de obra calificada y con visión futurista. Kim, S. S., \& Prideaux, B. (2006). 


\section{Relaciones internacionales}

Para Corea del norte ha sido complicado el tema de las relaciones internacionales, desde hace varias décadas han tenido sus altercados con Estados Unidos por la nuclearización y amenazas incluso contra su propio vecino del sur. George W. Bush en su momento de presidente catalogo a Corea del Norte como parte del grupo de "países de eje del mal". Corea del Norte es un país totalmente cerrado y tiene una doctrina de país autosuficiente. Lleno de conflictos a nivel internacional por el tema nuclear. Una política belicista así se podría definir a Corea del Norte con sus ideales siempre puesto en el armamento nuclear y dotaciones para su ejército Nacional. Insua, M. C. (2016).

La OEC por su parte en observaciones recientes encuentra que Corea del Norte no tiene una economía tan cerrada como todos pensaban, ha estado haciendo sus negocios tradicionales con su principal socio: China y esparciendo un mercado a través de la hulla, productos textiles y agrícolas: minerales donde incluso alcanzo a tener relaciones con algunos países de Latinoamérica como México y Chile. Jiménez, J. J. L. (2004). Licona Michel, Á., Barajas, C., \& Ayesa, C. (2013).

Corea del Sur por su parte es un país desarrollado, con un amplio grupo de aliados internacionales y socios comerciales los cuales lo han llevado a ser una de las mejores economías del mundo y ser un país sostenible brindándole una excelente calidad de vida a sus ciudadanos. Una nación enfocada en la globalización y con una visión comercial bien puesta. Existe un conflicto con su vecino del norte y todo esto por la retirada de acuerdos a la no nuclearización. Hille, E., Shahbaz, M., \& Moosa, I. (2019).

Como socios estratégicos tiene a Estados unidos y a China quien en primera instancia es socio comercial de Corea del Norte a pesar de ello el contexto mundial y los permanentes cambios en la industria lo llevó a tener relaciones comerciales con el país del sur, ocupando un papel fundamental.

\section{Reunificación de las Coreas}

Para la reunificación deben existir dos factores muy importantes, como lo son el diálogo y la cooperación. Entender más a Corea del Norte, por lo que han pasado, sus conflictos internos $\mathrm{y}$ su sistema político totalmente diferente. La educación unificatoria desarrolla un papel fundamental teniendo en cuenta que Corea del Norte quiere aliñar sus asperezas para darle solución a sus problemas internos y a sus crisis de nivel global. Álvarez, M. (2018).

Múltiples trabas, choque de ideologías y factores culturales no han dejado evolucionar un acercamiento productivo entre ambos países. No obstante, es de gran importancia los encuentros entre los líderes Kim-Jong-Un y Moon Jae-in algo que sirvió para calmar el ambiente y llevar una esperanza de unión entre las dos naciones. Yu, W. I. (2004).

El presidente de Corea del Sur, como lo ha publicado en vivo en el canal de televisión actualidad.RT.com el 16 de agosto del 2019, ha prometido buscar la reunificación de la península Corena en el año 2045, teniendo en cuenta; la desnuclearización y la unificación, cuando se cumplan un siglo de la segunda guerra Mundial, donde manifestó la siguiente frase que traemos a relucir en esta investigación como es "Esa posible Corea unificada se podría convertir en un gran mercado y una de las mayores economías que traería "paz y prosperidad a sí misma, al este de Asia y al mundo"

\section{Resultados}

Se comprendió desde el punto de vista como sociedad y como profesionales en comercio internacional este conflicto de gran magnitud que lleva más de 70 años y cómo ha afectado a sus habitantes en diferentes sectores como economía, política, cultura y comercio. Se definieron las consecuencias de este conflicto a nivel internacional y como aliados como Estados 
Unidos, Rusia y China cumplen un papel importante dentro de toda esta disputa y el rol de seguridad si mencionamos las problemáticas nucleares de Corea del norte.

\section{Discusión}

El comercio internacional de la península coreana está claramente a favor de los habitantes del sur donde tienen claro que el avanzar constantemente y tecnificar sus productos es la clave. El repunte de su economía es asombroso, productos no tangibles como software están aumentando claramente el PIB de esta nación donde su principal mercado es Estados Unidos y claramente tienen un espacio bien ganado en la industria tecnológica y automotriz por medio del impacto de sus reconocidas marcas. sin embargo, algunas políticas del líder del norte Kim Jong-UN para la exportación de minerales está funcionando en pequeña medida. Se desea poner fin a la tensión que se vive en el norte de corea y la única salida en un mundo totalmente globalizado y netamente competitivo es crear más alianzas comerciales, unirse a mas bloques donde logre ser participe.

Varios sectores del comercio son censurados por la República democrática de corea del norte, como lo son la industria cinematográfica y musical donde un estricto control por parte del gobierno decide que producciones llegan o son modificadas para la visualización o comercialización en la península. La tecnología dentro de corea del norte no es relevante ni fundamental para los ciudadanos en su cotidianidad pues es difícil adquirir un teléfono inteligente. La red de internet también está continuamente intervenida por el gobierno, no hay una libre expresión para los medios de prensa y comunicación; Es un secreto lo que pasa en el norte, pero lo que llama la atención es la necesidad de buscar un bloque comercial por parte de corea del norte para poder subsistir y mejorar su economía.

\section{Conclusión}

En el pasado artículo se trató el conflicto entre las dos coreas (Norte y sur) explicando desde sus orígenes por que se inició el conflicto y todo lo que ha conllevado esta península, convirtiéndola en la zona fronteriza más desigual del mundo donde sus políticas son dinámicas, su cultura totalmente diferente $\mathrm{y}$ un sistema económico de pasos agigantados sobre uno apenas se sostiene con un sistema socio-comunista; Las actitudes de adaptación, la salud, la convivencia son factores de análisis en el artículo además de la educación y su rol para la posible unificación de estas dos naciones las cuales en el panorama internacional son totalmente opuestas.

La afectación de este conflicto, las decisiones que se tomen y los sectores comerciales que son sensibles a esta problemática. Es incierto lo que pueda llegar a pasar en la península coreana, las decisiones nucleares son decisivas para la seguridad de Norteamérica y de Asia tanto como las vetas económicas. Dos mundos diferentes separados por una frontera, víctimas de un conflicto y un país que quiere salir de su crisis y buscar una mejora en su economía.

Referencias bibliográficas

Álvarez, M. (2018). Las dos Coreas: un cruel legado de la Guerra Fría. Revista Nueva Sociedad

Astor Molero, P. (2015). Comprender la península coreana: una historia de unión y división. Análisis histórico-comparativo de Corea del Norte y Corea del Sur.

Chiñas, C. G. (2003). Comercio exterior y desarrollo económico, el caso de Corea del Sur. Análisis económico, 18(37), 141-155.

Giné, J. (2008). Corea, una nación dividida en dos Estados. Política Exterior, 139-152.

Hille, E., Shahbaz, M., \& Moosa, I. (2019). The impact of FDI on regional air pollution in the Republic of Korea: ¿A way ahead to achieve the green growth strategy? Energy Economics, 81, 308-326.

Hyun, MK (2019). Determinantes del uso concurrente de biomedicina y medicina coreana: un estudio basado en la encuesta del Panel de 
Salud de Corea (2008-2014). European Journal of Integrative Medicine, 25, 1-5. Doi: $10.1016 /$ j. eujim.2018.11.005

Insua, M. C. (2016). Una guerra inolvidable: el conflicto de Corea y su cobertura mediática. Recuperado de http://cud. unizar. es/docum/17-\% 20 comunicacion $\%$ 20revisada. pdf.

Jiménez, J. J. L. (2004). Corea del Norte: ¿hacia la apertura y la reunificación con Corea del Sur? México y la Cuenca del Pacífico, (22), 40-45.

Kim, S. S., \& Prideaux, B. (2006). An investigation of the relationship between South Korean domestic public opinión, tourism development in North Korea and a role for tourism in promoting peace on the Korean península. Tourism Management, 27(1), 124-137.

Lee, M. W., \& Ahn, S. H. G. (2016). Relocation in space, language, and identity: Dislocated North Korean undergraduates in South Korean universities. Language \& Communication, 47, 43-52.

Licona Michel, Á., Barajas, C., \& Ayesa, C. (2013). Panorama de las relaciones ínter-coreanas en la era de Kim Jong-un. México y la Cuenca del Pacífico, 2(3), 75-101.

Liesa, F., Carlos, R., \& Borque Lafuente, E. (2013). El conflicto de Corea.

Min, W. (2004). El rol de la educación para la unificación de Corea. Comunicación escrita en Chile de cara al mundo asiático: Cultura y Negocios en APEC. Seminario de la Pontificia Universidad Católica de Chile. Santiago de Chile. Consulta on-line en: http://www7. uc. cl/ceauc/papers/Min_04.pdf.

Mora, J. P. (1998). La situación en la Península de Corea. Boletín de Información, (257), 3.
Park, S., Lee, M., Park, S. J., \& Lee, M. G. (2018). Health risk behaviors and psychological problems among South Korean, North Korean, and other multicultural family adolescents (2011-2016). Psychiatry research, 268, 373-380.

Shin, H., \& Stevens, Q. (2013). How Culture and Economy Meet in S outh Korea: The Politics of Cultural Economy in Culture-led Urban Regeneration. International journal of urban and regional research, 37(5), 1707-1723.

Training Society for Intercultural Education, $\&$ Research. International journal of intercultural relations: official publication of SIETAR: the Society for Intercultural Education, Training and Research. Elsevier Science Pergamon.

Yang, E. (2012). Corea del Norte en la encrucijada. Revista UNISCI, (30), 143-150.

Yu, W. I. (2004). Spatial Inertia of a Divided Nation: Korea, the Last Remnant of the Cold War. Petermanns geographische Mitteilungen, 148(5), 6-15. 